\title{
FÍSTULA PROSTATORECTAL YATROGÉNICA. REPARACIÓN CON COLGAJO PEDICULADO DE MÚSCULO GRACILIS. PRESENTACIÓN DE DOS CASOS
}

A. BLANCO DÍEZ, L. ÁlVAREZ CASTELO, E. FERNÁNDEZ ROSADO, Á. ÁLVAREZ JORGE*, M. RUIBAL MOLDES, S. NOVÁS CASTRO, A. BARBAGELATA LÓPEZ, M. GONZÁLEZ MARTÍN

Servicio de Urología. *Cirugía Plástica. Complejo Hospitalario Universitario Juan Canalejo. La Coruña.

Actas Urol Esp. 28 (6): 466-471, 2004

\section{RESUMEN}

FÍSTULA PROSTATORECTAL YATROGÉNICA. REPARACIÓN CON COLGAJO PEDICULADO DE MÚSCULO GRACILIS. PRESENTACIÓN DE DOS CASOS

Presentamos dos casos clínicos en los cuales durante la realización de una adenomectomía retropúbica por patología prostática benigna se produce una fístula prostatorectal. Describimos la cirugía de reparación de la misma con colgajo de músculo gracilis o recto interno, y su posterior buena evolución. Presentamos imágenes cistográficas y de enemas opacos que presentan un antes y un después de dicha cirugía. Los pacientes están sin incontinencia ni problemas relacionados con la cirugía de desinserción muscular.

PALABRAS CLAVE: Fístula prostatorectal. Músculo gracilis. Fistulorrafia. Complicaciones post-adenomectomía.

\section{ABSTRACT}

YATROGENIC PROSTATORECTAL FISTULA. USE OF GRACILIS MUSCLE PEDICLED FLAP FOR ITS REPARATION. PRESENTATION OF TWO CASES

We present two cases in which during retropubic prostatectomy for benign prostatic disease a prostatorectal fistula ocurred. We describe its reparation using a pedicled flap of gracilis muscle. We also present cistografic and opaque enema images wich shows the before and after of this surgery. Patients had good outcome without incontinence nor problems related to muscle desinsertion surgery.

KEY WORDS: Prostatorectal fistula. Gracilis muscle. Fistulorraphy. Complications post retropubic adenomectomy.

$\mathrm{L}^{2}$ as fístulas prostato-rectales y uretro-rectales son una rara consecuencia de la cirugía urológica y rectal, así como de la radiación pélvica y de las enfermedades inflamatorias intestinales. Representan un grave problema para el paciente, y para el cirujano debido a la dificultad técnica de dichas reparaciones y su tendencia a la recidiva. Existen varios métodos para su tratamiento, ninguno de ellos ha demostrado ser per- fecto. Presentamos dos casos clínicos en los que utilizamos el abordaje perineal y un colgajo pediculado de músculo gracilis, para la reparación de dos fístulas prostatorectales producidas tras cirugía por hiperplasia benigna de próstata (adenomectomía retropúbica). Los dos casos presentaron óptima evolución y en nuestra opinión ésta es una técnica segura para resolver este tipo de problemas. 


\section{CASOS CLÍNICOS}

Caso 1: Varón de 74 años. Adenomectomía retropúbica en abril 2001. Al día siguiente de la intervención se detecta rectorragia y tras exploración digital rectal se comprueba la existencia de una fistula prostato-rectal. Se realiza en primer lugar una colostomía y se coloca sonda vesical. Cistografía retrógrada y enema opaco: trayecto fistuloso uretro-rectal (Fig. 1).

Enero de 2002, tras persistencia de fístula se realiza cirugía: fistulorrafia por vía suprapúbica trans-prostática. Apertura de cápsula prostática y abordaje de fistula prostato-rectal. Fistulorrafia y sutura en dos planos ( $1^{\circ}$ rectal, $2^{\circ}$ prostático). Sonda vesical.
En posteriores controles, tras retirada de sonda, persiste fístula.

Abril 2002, electrofulguración de pequeño orificio fistuloso en celda prostática.

Mayo 2002. Enema opaco: paso de contraste de recto a vejiga.

Junio de 2002. Reparación de fístula con colgajo pediculado de músculo gracilis. Seguimiento: tras 13 meses de evolución, el paciente se encuentra asintomático, sin pérdidas de orina. Buena continencia. La cistografia y enema opaco de control muestran resolución de fístula prostato-rectal (Fig. 2). Por otra parte, no presenta problemas funcionales debidos a la desinserción muscular.
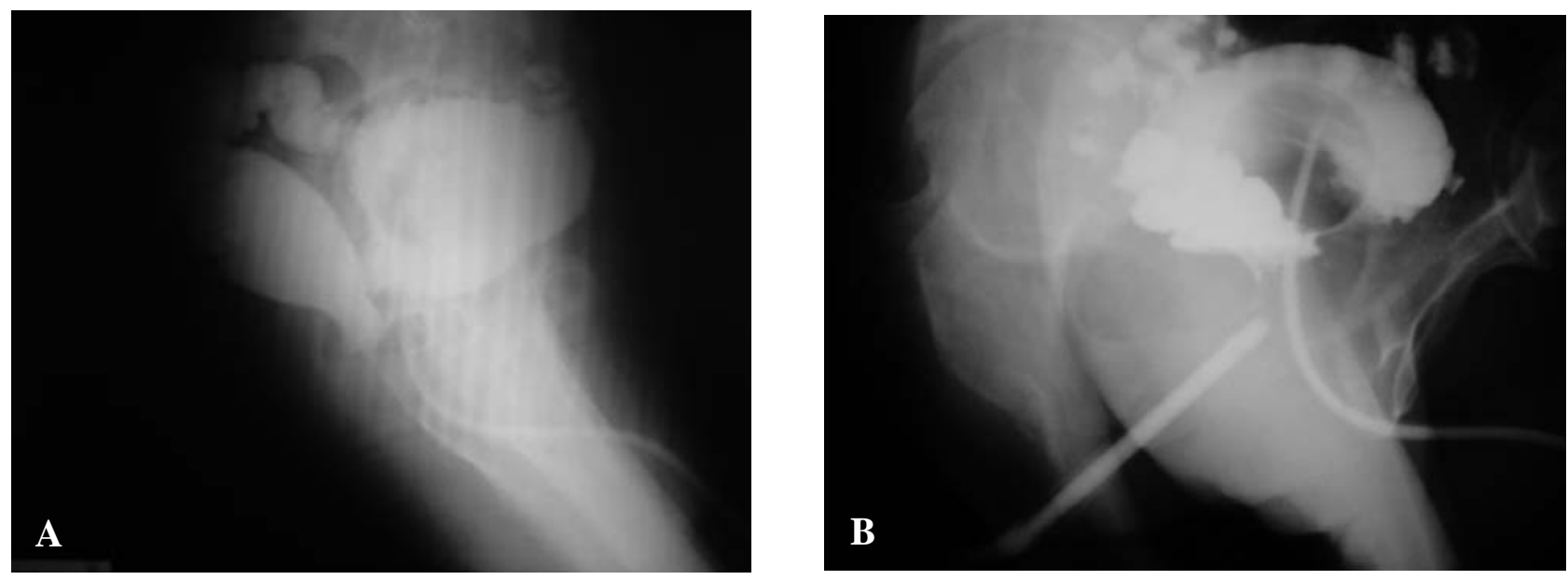

FIGURA 1. Cistografia A) y enema opaco B), que muestran importante fistula uretro rectal.
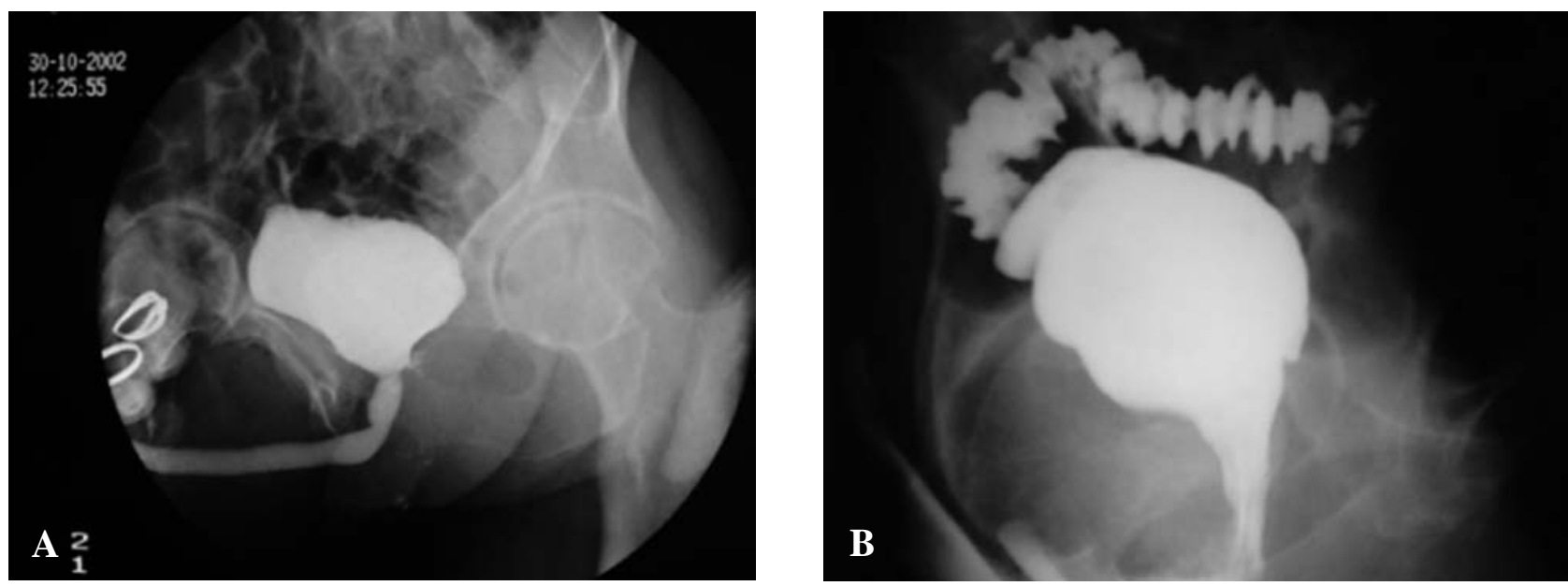

FIGURA 2. Cistografía y enema opaco post-intervención que muestran reparación total de la fistula con recuperación de anatomía de cuello vesical, celda prostática y tabique recto-prostático. 
Caso 2: Varón de 67 años. Adenomectomía retropúbica en abril de 2002 . Al $4^{\circ}$ día post-operatorio se constata fístula prostato-rectal, debido a que el paciente presenta rectorragias y al tacto rectal se palpa el balón de la sonda. Se instaura nutrición parenteral y tras mes y medio desaparecen las pérdidas. Cistografía al alta: no evidencia de fugas (mayo de 2002).

Seguimiento: el paciente refiere, meses más tarde, pérdidas de orina por recto. Tacto rectal: fístula próstato-rectal. Cistografía y cistoscopia: fistula persistente desde celda prostática alta (casi cuello vesical) hasta recto.

Cistografia: se observa fístula prostato-rectal (Fig. 3).

Enero 2003: Reparación de fístula con colgajo pediculado de gracilis.

Seguimiento: tras 6 meses el paciente refiere desaparición total de las pérdidas rectales, se encuentra asintomático, sin clínica de disuria ni pérdidas de orina. Asimismo no presenta problemas motores en relación a la cirugía de desinserción muscular. La cistografía muestra ausencia de fístula prostato-rectal (Fig. 4).

\section{Técnica quirúrgica}

Posición de litotomía forzada. Comprobación endoscópica de localización del orificio fistuloso prostático, tras inyección por sonda uretral de azul de metileno. Incisión en "U" o "media luna invertida" a $2 \mathrm{~cm}$ del margen anal. Disección con sección del tendón central y separación del esfín-

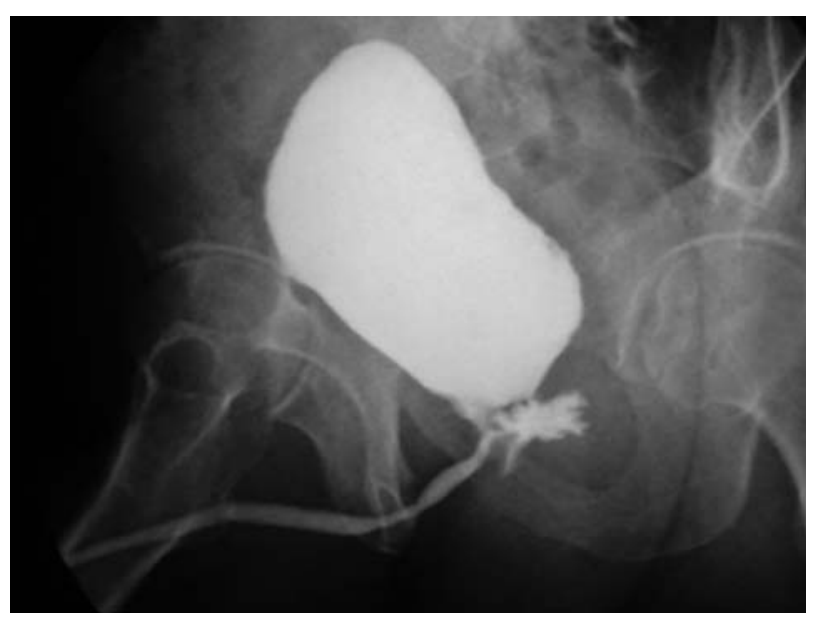

FIGURA 3. Cistografia que muestra existencia de pequeña comunicación fistulosa entre celda prostática y recto.

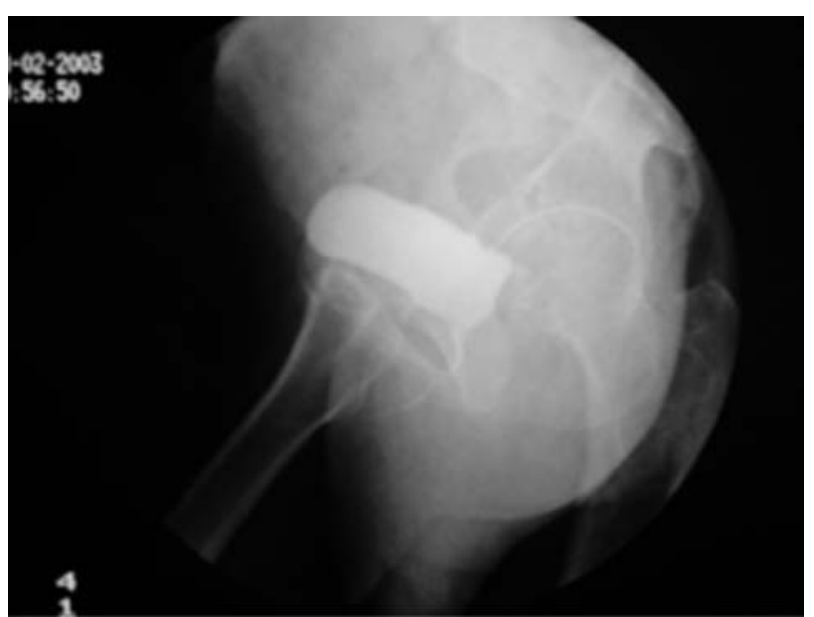

FIGURA 4. Cistografía post-intervención. Ausencia de fugas y reparación total del defecto.

ter anal, abordando espacio prerrectal. Disección progresando hasta abrir el orificio fistuloso, colocando una sonda de Foley en el orificio prostático y liberándolo. Se resecan bordes de fístula y se sutura en un plano. Se resecan bordes del orificio fistuloso rectal y se sutura en un plano horizontal.

En colaboración con el cirujano plástico, se diseca y libera el músculo recto interno (gracilis). Se secciona en su porción distal y se asciende pediculado y por túnel subcutáneo se interpone entre próstata y recto fijándolo en varios puntos.

Se colocan dos drenajes: penrose en periné y aspiración en muslo. Se deja sonda de Foley (Fig. 5).

\section{DISCUSIÓN}

Las fistulas prostato-rectales o uretro-rectales son una rara pero fatal consecuencia de la cirugía urinaria o rectal, o de los traumatismos. Pueden existir otras causas como las neoplasias pélvicas, la radiación o las enfermedades inflamatorias, por ejemplo la Enfermedad de Crohn. Son de difícil solución y representan un serio problema médico y de calidad de vida para el paciente. Pocos cirujanos tienen suficiente experiencia en el manejo de este problema, representando siempre un reto quirúrgico, debido a la dificultad de acceso al trayecto fistuloso y a su elevado índice de recurrencias. Debido a esta escasa frecuencia y a su dificultad técnica ningún procedimiento ha demostrado ser mejor que el resto. 


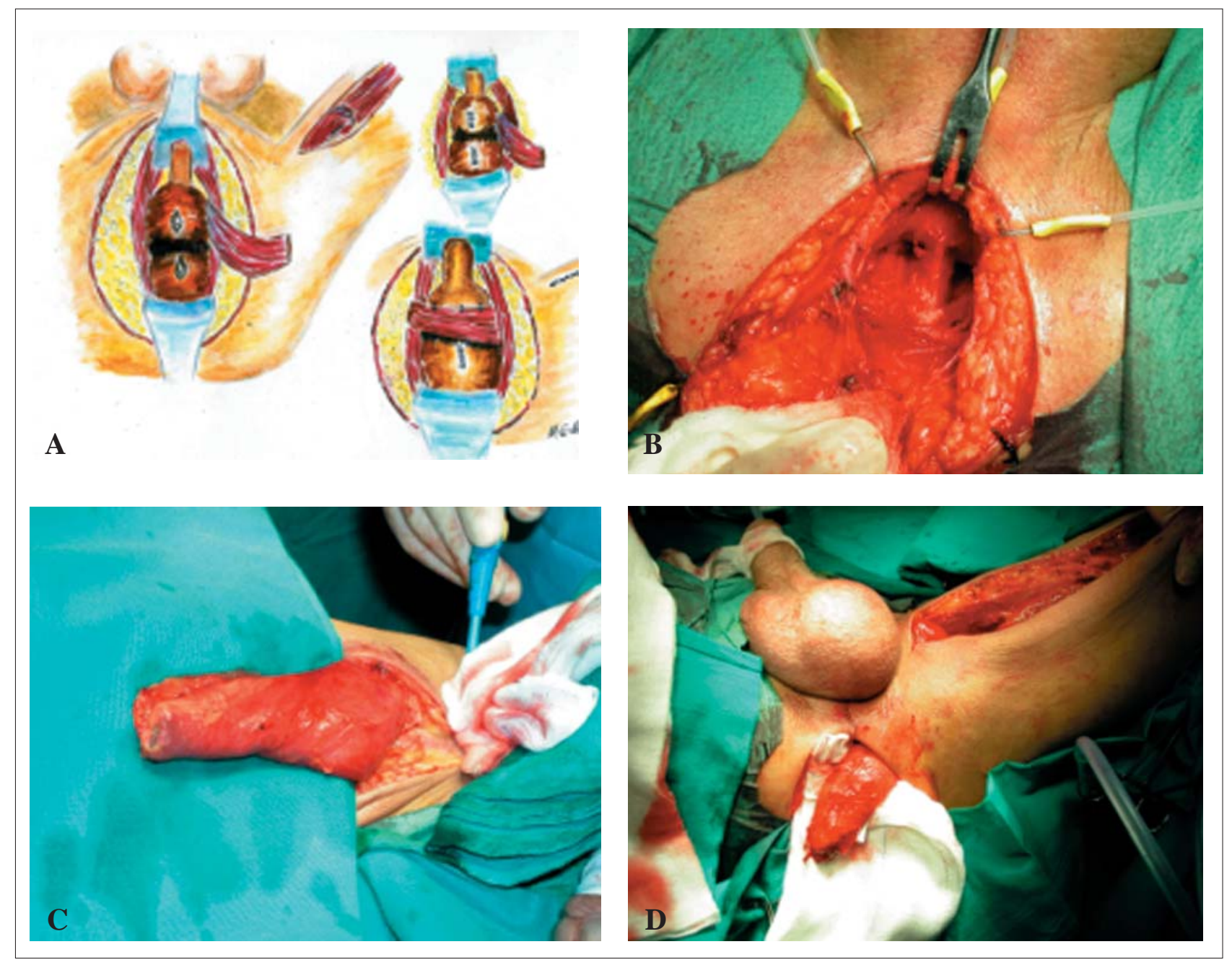

FIGURA 5. Esquema quirúrgico. A). Observamos diferentes pasos de la intervención desde la disección y exposición del trayecto fistuloso B), hasta la disección y liberación C), y transposición D) del músculo gracilis.

La causa más frecuente de fístula prostatorectal adquirida es el tratamiento de la patología prostática, sobre todo por radiación, lesión rectal intraoperatoria, o la instrumentación uretral ${ }^{1}$.

$\mathrm{El}$ manejo conservador inicial derivando heces y orina permite el cierre espontáneo de alguna de estas fístulas. Las que no curan en 3-6 meses necesitarán la cirugía para su resolución ${ }^{2}$. Aquellos pacientes no candidatos a cirugía podrían ser tratados con ciclos de antibióticos durante tiempo y permanecer libres de infecciones sistémicas ${ }^{3,6}$.

Existen varios métodos de reparación. Los más usados son:

Abordaje transabdominal: permite la utilización de un flap de omento para interponerlo en el trayecto fistuloso. Muchos cirujanos pueden estar cómodos en este campo, sin embargo pueden existir dificultades de maniobrabilidad debido a la mala exposición en la profundidad pélvica, sobre todo a la hora de cerrar el defecto uretral. Turner-Warwick recomienda en este abordaje dejar el defecto uretral abierto con un catéter fenestrado sobre el flap de omento ${ }^{4}$. Esta técnica tiene asociadas las complicaciones de toda la cirugía abdominal. Además de mayor tiempo post-operatorio puede ser difícil de realizar si existen adherencias de cirugias previas. No debemos olvidar complicaciones específicas de esta técnica como son la impotencia y la incontinencia por lesión de las ramas de los nervios pélvicos. Por todo esto, este abordaje apenas se usa hoy día, si exceptuamos las fístulas muy altas o las muy extensas ${ }^{1}$. 
Abordaje anterior transanorectal: consiste en una incisión perineal en la línea media profundizada incidiendo todas las estructuras superficiales a la cápsula prostática incluyendo la fascia perineal, el tendón central y el esfinter externo e interno ${ }^{5,7}$. Permite buen acceso para la reparación de estas fístulas, es un abordaje escasamente sangrante, tenemos tejido cerca (dartos, gracilis) para interposición, y ofrece un buen acceso a la uretra posterior en caso de estenosis. Es fundamental mantenerse siempre en la línea media para evitar la lesión de los nervios erectores.

Abordaje transanal: Popularizado por Parks y Motson en 1983, tiene las ventajas de su simplicidad, sin incisión y sin riesgo de infecciones de herida, pero permite poca exposición por lo que apenas se usa. Su indicación sería únicamente fistulas pequeñas cercanas al margen anal ${ }^{8,9}$.

Abordaje laterosacro de Kraske: Proporciona excelente exposición y no divide ni diseca el mecanismo esfinteriano anal. La gran desventaja es la necesidad de escindir 2 a 3 segmentos sacros, y por tanto sus músculos, nervios y ligamentos, existe el riesgo de incontinencia fecal por denervación, además de incontinencia urinaria. No se usa hoy día apenas ${ }^{2,10}$.

Abordaje posterior transesfintérico por línea media: conocido como de York-Mason ${ }^{1,2,11-13}$. Permite rápida exposición a través de áreas no cicatriciales, con mínimo sangrado. Una amplia serie presentada por Stephenson y Middleton, con 16 casos, obtiene unos resultados cuya base es: tiempo operatorio medio de 2 horas, pérdida sanguínea mínima (entre 50-400 cc), sin transfusiones. La estancia hospitalaria varía entre 2-11 días, siendo suficiente dos días en casos no complejos $^{12}$. Esta técnica evita las bandeletas neurovasculares, y resto de estructuras del suelo pélvico tan necesarias para la función sexual y la continencia. Ningún autor ha reportado recurrencia de la fístula con esta técnica. El temor de que la disección de los esfinteres rectales pudiese producir incontinencia fecal ha resultado infundado. La inervación de los esfinteres anales es posterolateral, por tanto el mantenerse siempre en la línea media y el cuidado en la aproximación a los esfinteres es la clave de la continencia ${ }^{1,2,13}$. Por otra parte cuando se realiza siguiendo los principios de la no superposición de suturas, y el uso de un flap avanzado de mucosa sus resultados son excelentes en grandes series ${ }^{12,14}$. Sin embargo, puede tener un valor limitado en grandes fístulas, cuando se hace necesario un colgajo pediculado debido a la ausencia de tejido adyacente utilizable. En cualquier caso esta es la técnica más usada en la literatura actual. El paciente se coloca en decúbito prono "posición de navaja", en extensión. Se incide desde el cóccix hasta el margen anal, por línea media, abriendo la pared posterior del recto y se incide el esfinter anal posterior de igual manera, con incisión limpia y marcando bordes con sutura para facilitar posterior reparación. Se accede a la pared anterior y fistula, más fácilmente identificable ésta si se coloca sonda al inicio de la intervención. Se reseca en su totalidad el trayecto fistuloso suturando por separado los planos prostático y rectal.

Abordaje perineal: Los dos casos que presentamos los realizamos con esta técnica. Tiene la gran ventaja de ser un campo muy bien conocido por el urólogo, habiendo recibido más atención en la literatura urológica que en la colorectal. Además de ser un campo conocido, permite una buena exposición, y tenemos a mano tejido adyacente para interponer (gracilis, dartos) ${ }^{15-19}$. La técnica, inicialmente descrita por Goodwin ${ }^{20}$, incluía la disección hasta la fístula en el mismo modo que lo describimos nosotros. Sin embargo una modificación que puede ser necesaria en ocasiones, ya que amplía enormemente el campo quirúrgico, sería la disección anterior en la línea media, del esfinter anal, similar al abordaje transesfintérico anterior. Esta modificación nos permite la reparación de complejas fístulas prostatorectales como no lo hace ninguna de las otras técnicas. De todas maneras el abordaje perineal tiene algún inconveniente. Por una parte atraviesa tejidos cicatriciales de difícil disección y existe si no se hace con cuidado, elevado riesgo de impotencia. En amplias revisiones se ha encontrado algún caso de recurrencia y de incontinen$\mathrm{cia}^{2}$. En cualquier caso el correcto conocimiento de la anatomía debe evitar esto último, como siempre lo más importante es el no abandono de la línea media, evitando disecciones laterales en la exposición del trayecto fistuloso ${ }^{1}$. Los principios básicos de esta cirugía, ya propuestos por Goodwin en $1958^{20}$, y que nosotros seguimos en 
estas intervenciones, son: escisión de la fístula, dejar amplias zonas de tejido sano en los lados uretroprostático y rectal, y cierre de ambos lados de la fístula por separado y con suturas no superpuestas. Como en nuestro caso, algunos cirujanos prefieren la interposición entre los dos segmentos, de tejido sano bien vascularizado, que de más seguridad a la reparación. Los más usados son el dar$\operatorname{tos}^{15,16}$, y el gracilis ${ }^{17-19}$, en otras ocasiones el omento (que requiere abordaje combinado abdominal), o colgajos pediculados de ingle ${ }^{15}$. En nuestro caso nos pareció el gracilis el más indicado, ya que es un músculo secundario, que no realiza funciones motoras principales, que es fácilmente accesible para su desinserción, y que no deja secuelas importantes. Además tiene un pedículo vascular largo que permite su correcta vascularización. Este músculo es frecuentemente usado por los cirujanos plásticos para reparaciones diversas, y cumple los criterios para este tipo de reparaciones.

Esta técnica nos ha resultado muy útil para la solución definitiva de estos casos. La familiaridad con el campo permitió una buena exposición para la identificación, disección, escisión y reparación de las dos fistulas. No fue necesario el abordaje abdominal, ni la cirugía anorrectal, y los resultados posteriores han sido excelentes, con mínimas consecuencias funcionales para los pacientes.

Para concluir, baste un concepto claro: el primer intento de reparación de una fístula uretro, o prostato-rectal, debe ser siempre el definitivo. La recurrencia después de una cirugía infructuosa aumenta la fibrosis alrededor de la zona, haciendo que los sucesivos intentos de reparación sean cada vez más difíciles. Nosotros creemos que el abordaje perineal con interposición de tejido muscular pediculado bien vascularizado, ha demostrado ser una técnica con muchas ventajas y nos parece la más indicada para estos pacientes.

\section{REFERENCIAS}

1. VISSER BC, MC ANINCH JW, WELTON ML.: Rectourethral fistulae: the perineal approach. $\mathrm{J} \mathrm{Am}$ Coll Surg 2002; 195: 138-143.

2. BUKOWSKI TP, CHAKRABARTY A, POWELL IJ, FRONTERA R, PERLMUTTER AD, MONTIE JE.: Acquired rectourethral fistula: methods of repair. $J$ Urol 1995; 153: 730-733.

3. THOMPSON IM, MARX AC.: Conservative therapy of rectourethral fistula. Five-year follow-up. Urology 1990; 35: 533-536.
4. TURNER-WARWICK R.: The use of pedicle grafts in the repair of urinary tract fistulae. $\mathrm{Br} J$ Urol 1972; 44: 644-647.

5. GECELTER L.: Transanorectal approach to the posterior urethra and bladder neck. J Urol 1973; 109: 1011-1016.

6. LÓPEZ LÓPEZ C, ROMERO MAROTO J, GÓMEZ RUIZ JJ.: The acquired urethrorectal fistula: a rare pathology. Arch Esp Urol 1995; 48: 339-342.

7. CHTOUROU M, KHALFALLAH T, DAHMOUL H, HORCHANI A.: Anterior anal sphincter approach in the management of urethral-rectal fistulas: a case report. Prog Urol 1997; 7: 640-642.

8. PARKS AG, MOTSON RW.: Perianal repair of rectoprostatic fistula. Br J Surg 1970; 70: 725-727.

9. HATA F, YASOSHIMA T, KITAGAWA $S$ et al.: Transanal repair of rectourethral fistula alter a radical retropubic prostatectomy: report of a case. Surg Today 2002; 32: 170-173.

10. SWEENEY WB, DESHMUKH N.: Modified Kraske approach for disease of the mid-rectum. Amer $J$ Gastroenterol 1991; 86: 75-77.

11. YORK MASON A.: Surgical access to the rectum- a transesphincteric exposure. Proc Roy Soc Med 1970; 63: 91-94.

12. STEPHENSON RA, MIDDLETON RG.: Repair of rectourinary fistulas using a posterior sagittal transanal transrectal (modified York-Mason) approach: an update. J Urol 1996; 155: 1989-1991.

13. BOUSHEY RP, Mc LEOD RS, COHEN Z.: Surgical management of acquired rectourethral fistula, emphasizing the posterior approach. Can J Surg 1998; 41: 241-244.

14. AL-ALI M, KASHMOULA D, SAOUD IJ.: Experience with 30 postraumatic rectourethral fistulas: presentation of posterior transsphincteric anterior rectal wall advancement. J Urol 1997; 158: 421-424.

15. YOUSSEF A-H, FATH-ALLA M, EL-KASSABY AW.: Perineal subcutaneous dartos pedicled flap as a new technique for repairing urethrorectal fistula. $J$ Urol 1999; 161: 1498-1500.

16. YAMAZAKI Y, YAGO R, TOMA H.: Dartos flan interposition in the surgical repair of rectourethral fistulae. Int J Urol 2001; 8: 564-567.

17. RYAN JA Jr, GIBBONS RP, CORREA RJ Jr.: Urologic use of gracilis muscle flap for nonhealing perineal wounds and fistulas. Urology 1985; 26: 456-459.

18. ZMORA O, POTENTI FM, WEXNER SD et al. Gracilis muscle transposition for iatrogenic rectourethral fistula. Ann Surg 2003; 237: 483-487.

19. RIUS J, NESSIM A, NOGUERAS JJ, WEXNER SD.: Gracilis transposition in complicated perianal fistula and unhealed perineal wounds in Crohn's disease. Eur J Surg 2000; 166: 218-222.

20. GOODWIN WE, TURNER RD, WINTER CC.: Rectourinary fistula: principles of management and technique of surgical closure. J Urol 1958; 80: 246254.

Dr. A. Blanco Díez

C/ Montserrat, $18-6^{\circ} \mathrm{E}$

15009 La Coruña

(Trabajo recibido el 2 septiembre de 2003) 